\title{
EFEITO DO UMEDECIMENTO E DA VELOCIDADE DE DEFORMAÇÃO SOBRE A RESISTÊNCIA DE SOLOS CIMENTADOS ARTIFICIALMENTE
}

\author{
Henrique Gonçalves Carvalho Júnior \\ Instituto Federal de Educação, Ciência e Tecnologia do Rio Grande do Norte \\ Graduando em Tecnologia em Construção de Edifícios e Bolsista do PIBITI-CNPq \\ Fone: 8488242919 \\ henriquejunior_5@hotmail.com \\ Dárcia Sâmia Santos Moura \\ Instituto Federal de Educação, Ciência e Tecnologia do Rio Grande do Norte \\ darciasamia@hotmail.com \\ Ricardo Nascimento Flores Severo \\ Instituto Federal de Educação, Ciência e Tecnologia do Rio Grande do Norte \\ Orientador em bolsa do PIBITI-CNPq \\ ricardoflores@cefetrn.br \\ Olavo Francisco dos Santos Júnior \\ Universidade Federal do Rio Grande do Norte \\ olavo@ct.ufrn.br \\ Ovídio Cabral de Macedo Neto \\ Instituto Federal de Educação, Ciência e Tecnologia do Rio Grande do Norte \\ ovidiocabral@hotmail.com \\ Régia Lúcia Lopes \\ Instituto Federal de Educação, Ciência e Tecnologia do Rio Grande do Norte \\ regia@cefetrn.br
}

\begin{abstract}
RESUMO
Este trabalho apresenta e analisa dados de ensaios de resistência à compressão simples realizados em um solo laterítico cimentado artificialmente. O solo usado foi classificado pelo SUCS como SC (areia argilosa). No trabalho foram consideradas quatro variáveis: teor de cimento ( $2 \%, 5 \%$ e $10 \%)$, densidade (três energias distintas de compactação), efeito de umedecimento (amostras inundadas e não inundadas) e velocidade de deformação (0,01 e 1,0 $\mathrm{mm} / \mathrm{min}$ ). Os resultados mostraram que a resistência aumenta com o aumento do teor de cimento; diminui exponencialmente com o aumento do índice de vazios, a inundação provoca perda de resistência no solo apenas para teores de cimentos mais baixos e ocorre uma tendência de aumento da resistência em função do aumento da velocidade de deformação para amostras na condição mais compacta, esse aumento de velocidade é compatível com o observado em solos residuais e sedimentares com algum grau de cimentação.
\end{abstract}

PALAVRAS-CHAVE: Umedecimento, Solos Cimentados, Resistência à Compressão, Velocidade de Deformação. 


\title{
EFFECT OF THE WETTING AND THE RATE OF DEFORMATION ON THE SOIL STRENGT CEMENTED ARTIFICIALLY
}

\begin{abstract}
This work presents and analyses results of unconfined compression tests carried out in specimens of an artificially cemented lateritc soil. The soil used in this study was classified as SC (Clayey Sand). In the work four variables had been considered: amount of cement (2\%, $5 \%$ and $10 \%$ ), density (three distinct energies of compacting), effect of wetting (soaking and not soaking samples) and rate of deformation $(0.01$ and $1.0 \mathrm{~mm} / \mathrm{min})$. The results had shown that the strength increases with the increase of the cement content and decrease exponentially with the increase of the void ratio, soaking provokes loss of strength in the soil only for lower cement content and occurs a trend of increase of the resistance in function of the increase of the rate of deformation for samples in the condition most compact, This increase in rate of deformation is consistent with that seen in sedimentary and residual soils with some degree of cementation.
\end{abstract}

KEY-WORDS: Wetting, Cemented soil, Compressive strength, Rate of deformation.

Os resultados apresentados confirmam o esperado. 


\section{EFEITO DO UMEDECIMENTO E DA VELOCIDADE DE DEFORMAÇÃO SOBRE A RESISTENNCIA DE SOLOS CIMENTADOS ARTIFICIALMENTE}

\section{INTRODUÇÃO}

Vários estudos a respeito de solos cimentados ou misturas de solo-cimento vem sendo realizados com vistas a compreender o efeito das ligações entre as partículas sobre o comportamento mecânico desses solos. Solos com cimentação entre as partículas podem ser formados por diversos processos naturais, assim como, freqüentemente adiciona-se cimento ao solo para melhorar as suas propriedades geotécnicas. Dessa forma, é importante o conhecimento do efeito da cimentação entre as partículas do solo e os demais fatores que afetam o comportamento de solos cimentados, tanto para solos cimentados naturalmente como para solos com cimentação artificial.

Trabalhos anteriores indicam que a resistência do solo aumenta com o aumento do teor de cimento (Schnaid et al, 2001), com a diminuição do índice de vazios (Lo e Wardani, 2002; Consoli et al, 2007) e para um dado teor de cimento a resistência aumenta com a velocidade de deformação (Clough et al, 1981).

Neste trabalho estudou-se amostras cimentadas artificialmente a fim de analisar o efeito da intensidade da cimentação sobre o comportamento mecânico dos solos. Foram consideradas ainda como variáveis do estudo a densidade da mistura de solo-cimento, a inundação (umedecimento) dos corpos de prova antes da ruptura para reduzir o efeito da sucção e a velocidade de deformação. O solo empregado foi proveniente da Formação Barreiras, coletado na região costeira do Estado do Rio Grande do Norte, no município de Tibau do Sul, na região Nordeste do Brasil.

Estudos realizados nessa área indicam que a cimentação entre as partículas desenvolve influência positiva na estabilidade de taludes que formam falésias, em muitos casos, praticamente verticais na região (Silva 2003; Santos Júnior et al. 2004; Santos Júnior et al. 2005; Severo 2005). No entanto, a quantificação dessa influência ainda necessita de estudos detalhados.

O objetivo central do trabalho é analisar o efeito do umedecimento, do grau de cimentação teor de cimento) e da influência da velocidade de deformação sobre o comportamento de solos cimentados artificialmente, através da comparação dos resultados de ensaios de compressão simples realizados sobre amostras inundadas e não inundadas. 


\section{METODOLOGIA}

Utilizou-se neste trabalho um solo laterítico da Formação Barreiras, situada no município de Tibau do Sul, no estado do Rio Grande do Norte. O solo apresenta $88 \%$ de areia, $8 \%$ de silte e $4 \%$ de argila. Possui limite de liquidez de $28 \%$ e índice de plasticidade de $7 \%$. A densidade dos sólidos Gs é igual a 2,67. No Sistema Unificado de Classificação dos Solos, foi classificado como SC (areia argilosa). Na Figura 1 é apresentada a curva granulométrica do solo utilizado na pesquisa.

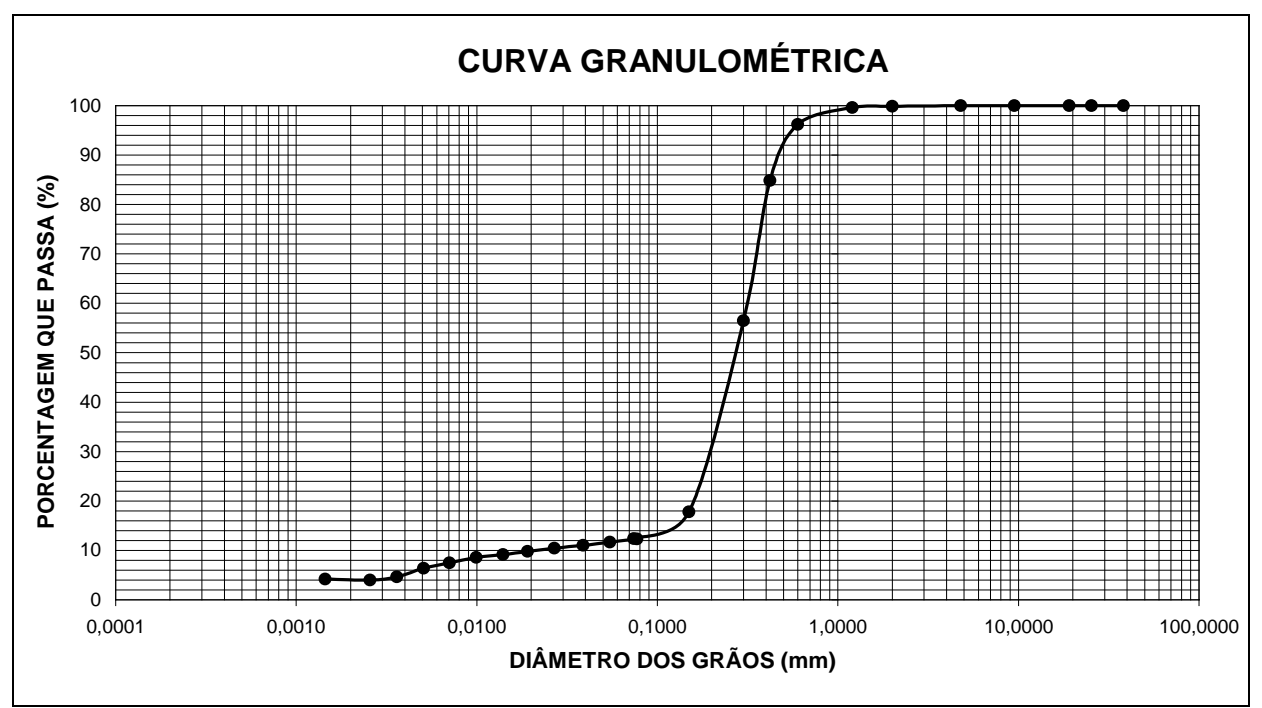

Figura 1. Curva granulométrica do solo.

A água utilizada nos ensaios foi fornecida pela Companhia de Água e Esgotos do Rio Grande do Norte (CAERN), com todas as suas características fisico-químicas dentro das normas brasileiras.

O cimento utilizado foi do tipo CP II - 32, com suas características físicas e químicas dentro das normas brasileiras (NBR 11.578/1991 - Cimento Portland Composto). O cimento ficou perfeitamente acondicionado em sacos plásticos de 1,0 kg fechados até as datas em que foram realizadas as respectivas moldagens.

As amostras de solo-cimento foram compactadas em moldes bi-partidos (provetes) metálicos cilíndricos com $10 \mathrm{~cm}$ de altura e $5 \mathrm{~cm}$ de diâmetro, em quatro camadas de $100 \mathrm{~g}$ cada, por meio da aplicação de golpes de um soquete metálico circular padronizado com massa de 400 gramas e área de $1,13 \mathrm{~cm}^{2}$, com três energias de compactação distintas (10, 30 e 60 golpes do soquete por camada).

Os Corpos de prova foram moldados com a quantidade constante de $20 \mathrm{ml}$ de água, ou seja, 5\% de água em relação ao peso do solo seco. A quantidade de água foi definida por ser a menor porcentagem que tornou possível a moldagem dos corpos de prova.

Quantidades menores de água provocaram a ruptura das amostras durante a desmoldagem, enquanto quantidades maiores permitem a desmoldagem, no entanto, elevam o fator água/cimento. Desta forma, poderiam diminuir a resistência mecânica, embora Consoli et al (2007), tenha verificado que a resistência é afetada de forma mais pronunciada pela densidade do que pelo fator água/cimento. 
As amostras foram curadas no ambiente do laboratório sob uma temperatura média de $25^{\circ} \mathrm{C} \mathrm{e}$ umidade do ar de $65 \%$ durante 28 dias.

Foram realizados ensaios de resistência à compressão simples, em amostras no teor de umidade pós-cura do solo e ensaios na condição inundada. Nestes últimos, as amostras foram submersas 72 horas antes do ensaio de resistência a compressão.

Considera-se teor de umidade pós-cura $\left(\mathrm{W}_{\mathrm{pc}}\right)$ àquele que a amostra se encontrava após o período de cura, obtida logo após a ruptura. O teor de umidade pós-cura foi menor do que $1 \%$ em todas as amostras. Este valor de umidade é devido ao fato da cura te sido realizada no ambiente de laboratório.

O teor de umidade na condição inundada variou entre $11 \%$ e $24 \%$ dependendo do grau de compactação dos corpos de prova. As amostras mais compactas apresentaram menores valores de teor de umidade.

Decorrido o tempo de cura, 46 corpos de prova (CPs), 23 na condição inundada e 23 na condição não inundada (umidade pós-cura), com os três teores de cimento citados, foram rompidos em uma prensa com velocidade de $0,5 \mathrm{~mm} /$ minuto, para analisar o efeito do umedecimento sobre a resistência mecânica dos solos e também a influência do grau de cimentação (teor de cimento). A seguir foram rompidos 16 corpos de prova, com teor de cimento constante de 5\%; oito rompidos com velocidade de $0,01 \mathrm{~mm} /$ minuto e oito com velocidade de $1,00 \mathrm{~mm} /$ minuto, para analisar o efeito da velocidade de deformação na resistência mecânica. Na Figura 2 é mostrada a seqüência de atividades para realização dos ensaios.

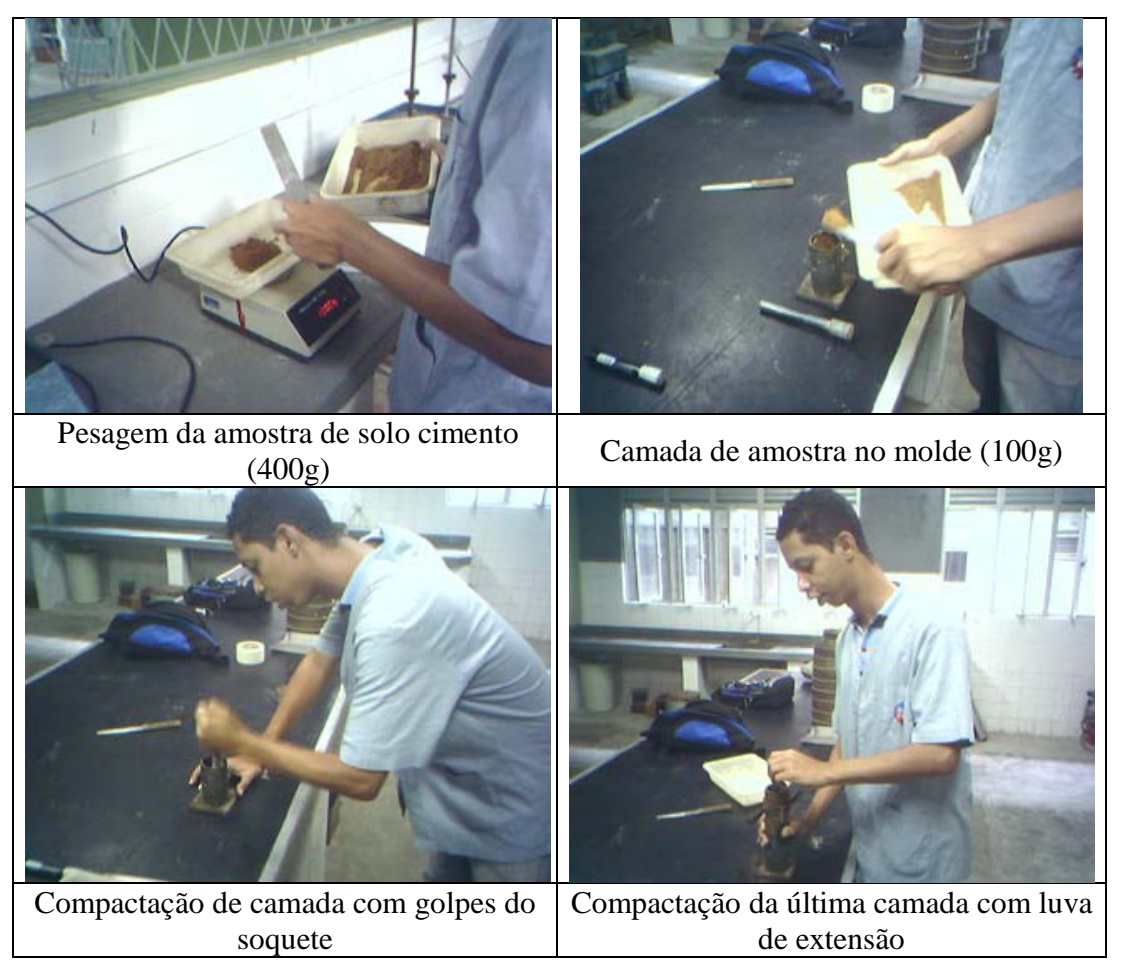




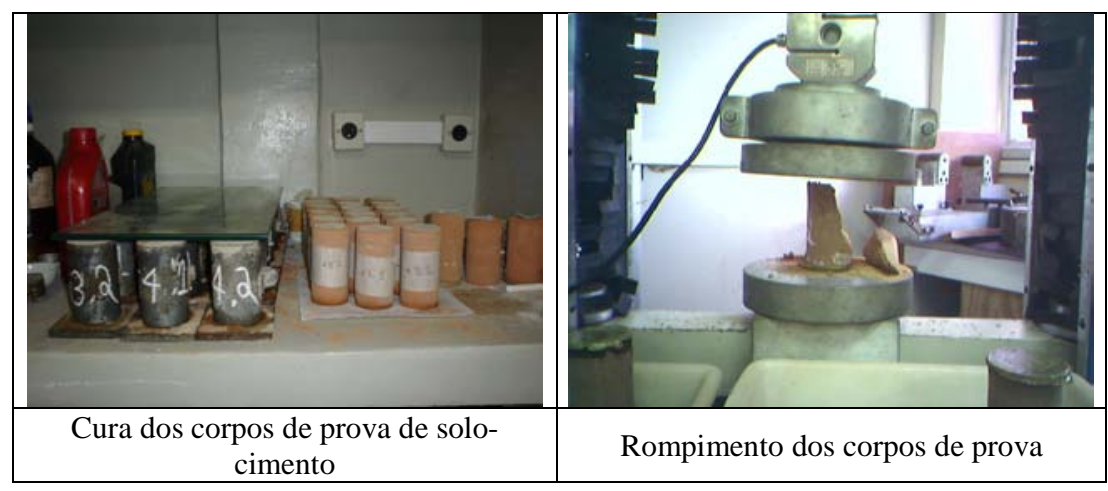

Figura 2. Metodologia de execução dos ensaios.

\section{APRESENTAÇÃO DOS RESULTADOS}

São apresentadas a seguir considerações a respeito do efeito do umedecimento sobre a resistência a compressão do solo em amostras com diferentes teores de cimento.

\section{Teor de cimento: $2 \%$}

No gráfico da Figura 3 é mostrado o efeito do umedecimento sobre a resistência à compressão não confinada para 17 (dezessete) amostras moldadas com 2\% de cimento. Verifica-se que a resistência diminui com o aumento do índice de vazios do solo tanto para as amostras inundadas como para as amostras secas. Este fato, mostra que ocorre um aumento na resistência com o aumento da intensidade de compactação. As amostras inundadas apresentam resistência inferior àquelas obtidas nos ensaios no teor de umidade natural, ou seja, a diminuição da sucção provocada pela inundação das amostras provoca redução da resistência ao cisalhamento. A linha de tendência que melhor se adapta aos resultados é a exponencial. Quantas amostras foram testadas?

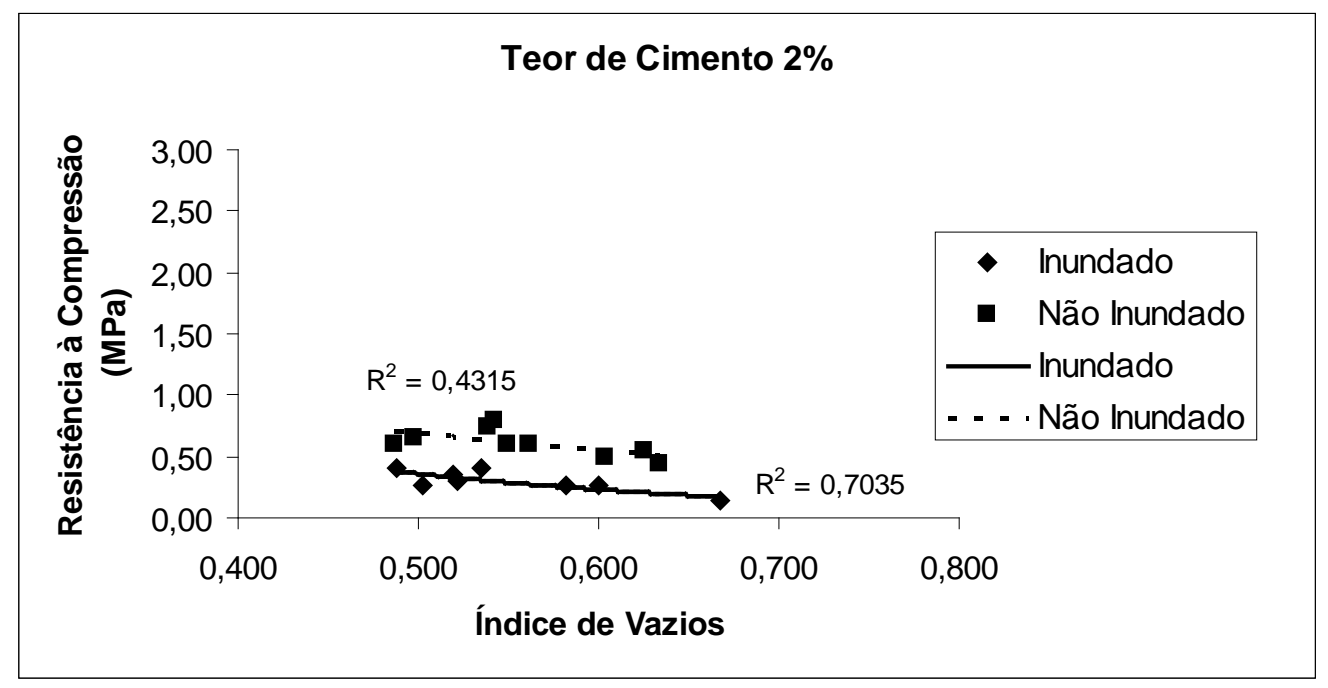

Figura 3. Resistência à compressão $x$ índice de vazios para amostras com teor de cimento de $2 \%$. 
Teor de cimento: $5 \%$

No gráfico da Figura 4 é mostrado o efeito do umedecimento sobre a resistência à compressão não confinada para 17 (dezessete) amostras com 5\% de cimento.

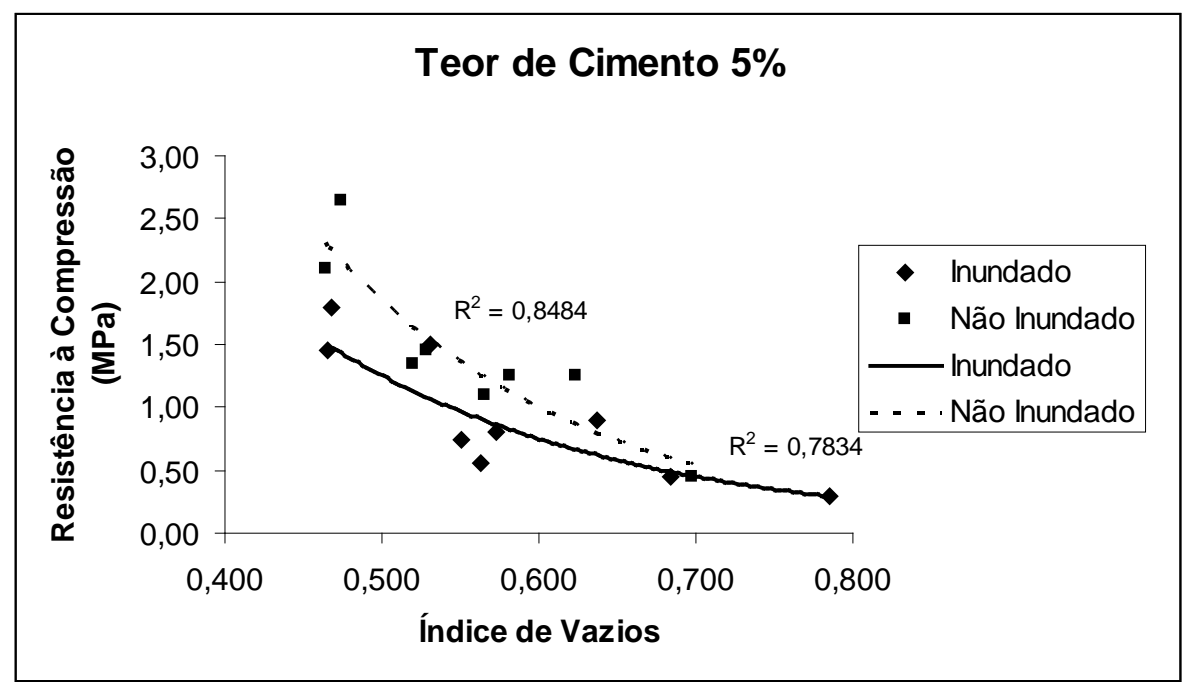

Figura 4. Resistência à compressão $\mathrm{x}$ índice de vazios para amostras com teor de cimento de $5 \%$.

Os resultados com 5\% de cimento mostraram uma tendência mais efetiva da redução da resistência com o aumento do índice de vazios comparativamente com os mostrados nas amostras com $2 \%$ de cimento. Por outro lado, o umedecimento parece afetar menos a resistência dos solos com 5\% de cimento, principalmente para valores mais altos de índice de vazios. Comparando-se os valores de R2 obtidos nas correlações mostradas nas Figuras 3 e 4, verifica-se uma acentuada melhora no coeficiente de correlação das amostras não inundadas, quando do aumento do teor de cimento de $2 \%(\mathrm{R} 2=0,43)$ para $5 \%(\mathrm{R} 2=0,85)$.

Teor de cimento: $10 \%$

No gráfico da Figura 5 é mostrado o efeito do umedecimento sobre a resistência à compressão não confinada para 12 (doze) amostras moldadas com 10\% de cimento.

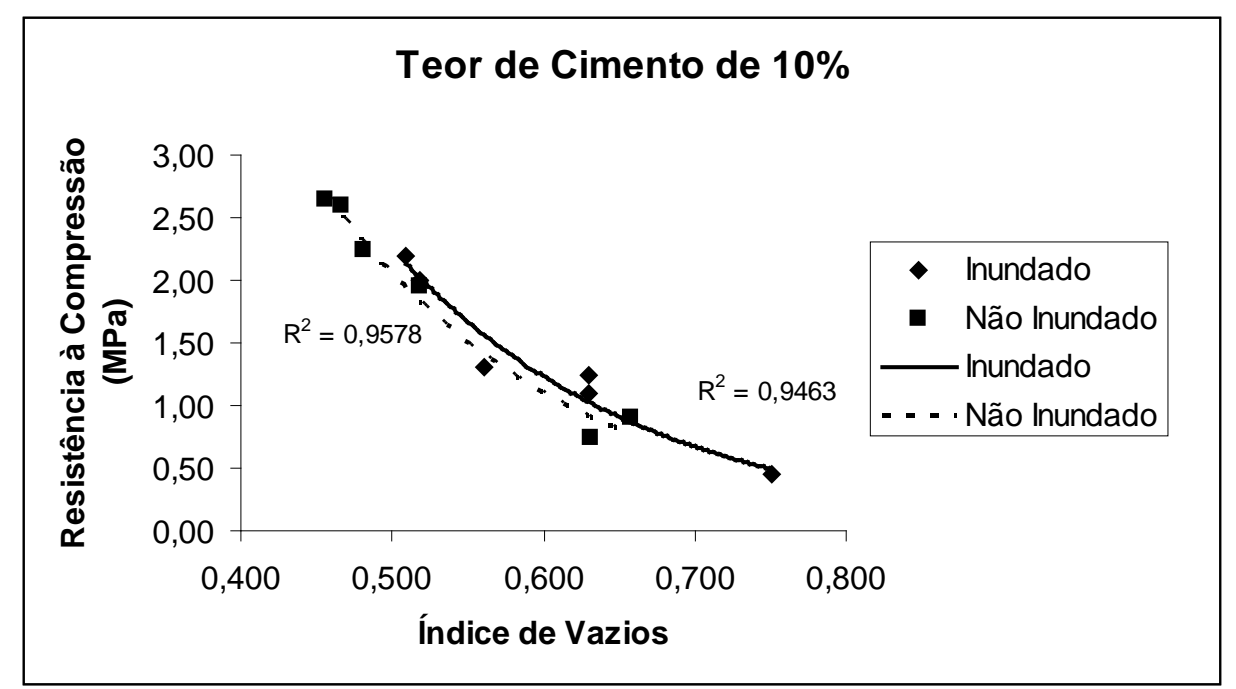

Figura 5. Resistência à compressão $x$ índice de vazios para amostras com teor de cimento de $10 \%$. 
O solo cimentado artificialmente com $10 \%$ de cimento apresentou aumento da resistência com o aumento da energia de compactação, de forma similar ao observado nas amostras com $2 \%$ e $5 \%$ de cimento. Conforme pode ser observado na Figura 5 as linhas de tendência exponenciais do solo inundado e não inundado estão muito próximas (uma da outra).

Verifica-se dessa forma que a inundação não afetou de maneira significativa a resistência à compressão das amostras preparadas com $10 \%$ de cimento. Ao se comparar os gráficos das Figuras 3, 4 e 5 constata-se que as linhas de tendência para as condições inundada e não inundada se aproximam com o aumento do teor de cimento.

Este fato mostra que o umedecimento afeta de forma mais pronunciada os solos com menores teores de cimento. No caso da Figura 5 (amostras com $10 \%$ de teor de cimento) o efeito da cimentação parece ser mais importante que o do umedecimento no comportamento das amostras. Observa-se mais uma vez o aumento no valor de $\mathrm{R}^{2}$ para as amostras com $10 \%$ de cimento, o que indica que a dispersão dos dados diminui com o aumento do teor de cimento.

São apresentadas a seguir considerações a respeito do efeito do teor de cimento sobre a resistência a compressão do solo em amostras com diferentes teores de cimento.

\section{Amostras inundadas}

No gráfico da Figura 6 é mostrado o efeito da intensidade de compactação, através do índice de vazios, sobre a resistência à compressão não confinada para as amostras moldadas com 2, 5 e $10 \%$ de cimento, na condição inundada.

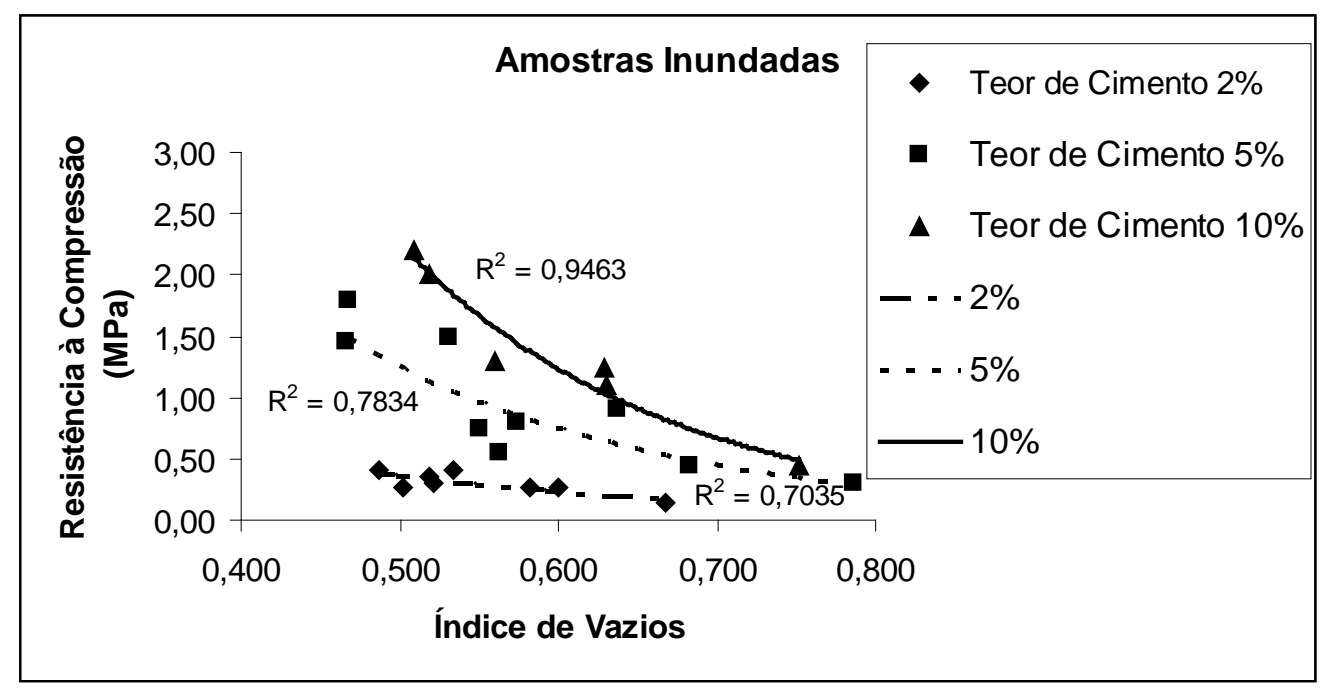

Figura 6. Resistência à compressão $x$ índice de vazios para amostras na condição inundada.

A análise da Figura 6 mostra que a resistência diminui com o aumento do índice de vazios, ou seja, a resistência aumenta com o aumento da energia de compactação. Entretanto, esse aumento é mais expressivo para valores mais elevados de teor de cimento. Para um dado índice de vazios (ou energia de compactação), a resistência aumenta com o aumento do teor de cimento. No entanto, pode-se observar na Figura 6 que para elevados valores de índice de vazios (ou baixas energias de compactação) o teor de cimento exerce pouca influência. 


\section{Amostras não inundadas}

No gráfico da Figura 7 é mostrado o efeito da intensidade de compactação, através do índice de vazios, sobre a resistência à compressão não confinada para as amostras moldadas com 2, 5 e $10 \%$ de cimento na condição não inundada.

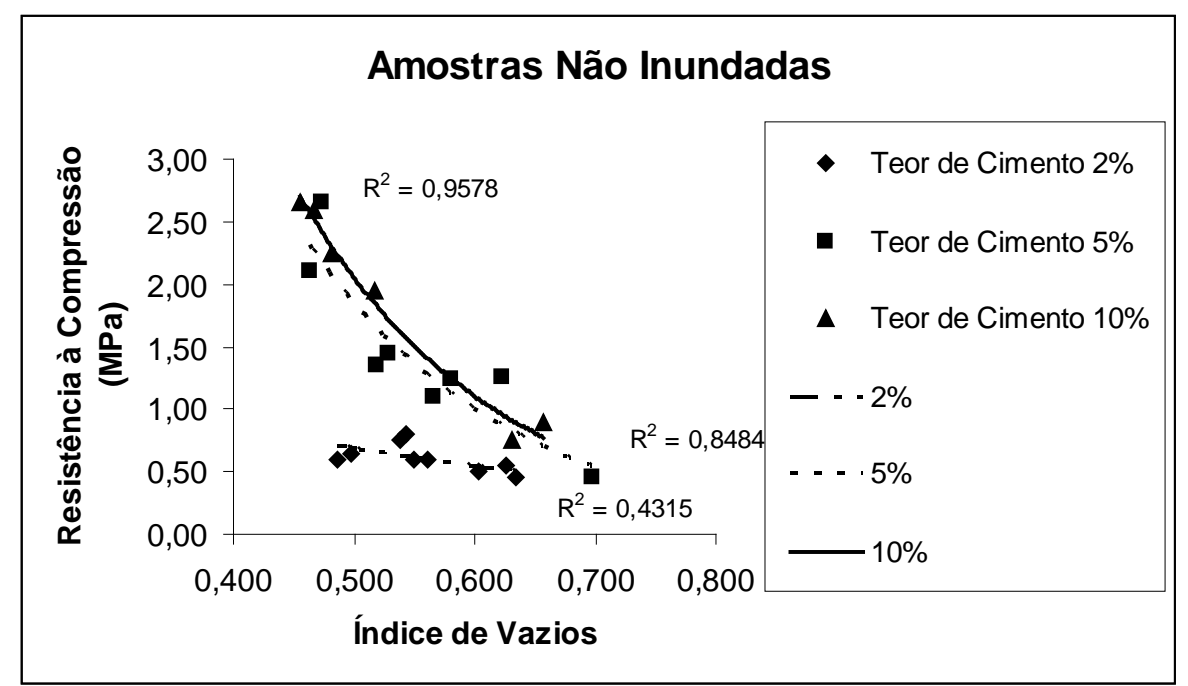

Figura 7. Resistência à compressão $x$ índice de vazios para amostras na condição não inundada.

Os resultados na condição não inundada mostram que os valores de resistência à compressão simples obtidos apresentam a mesma tendência das amostras na condição inundada, ou seja, a resistência diminui exponencialmente com o aumento do índice de vazios. No entanto, para as amostras moldadas com 5\% e 10\% de teor de cimento a resistência resultante foi da mesma ordem de grandeza, conforme pode ser observado pela proximidade das respectivas linhas de tendência na Figura 7.

De maneira geral os resultados obtidos em termos de variação de resistência com aumento da massa específica estão de acordo com a observação de Consoli et al (2007), segundo o qual a resistência aumenta exponencialmente com a redução da porosidade da mistura compactada.

São apresentadas a seguir considerações a respeito do efeito da velocidade de deformação sobre a resistência a compressão do solo em amostras com diferentes teores de cimento.

Foram realizados 16 (dezesseis) ensaios de compressão simples com o objetivo de avaliar a influência da velocidade de deformação, sendo oito ensaios realizados com a velocidade de $0,01 \mathrm{~mm} /$ minuto e oito com $1,0 \mathrm{~mm} /$ minuto. A NBR 12.025 que trata de ensaios de compressão simples de corpos-de-prova cilíndricos em solo-cimento, estabelece uma velocidade de deformação para ruptura de corpos de prova de solo-cimento de $1,14 \mathrm{~mm} / \mathrm{min}$, porém neste trabalho optou-se pelas velocidades referidas para analisar o efeito de um incremento de cem vezes na velocidade de deformação.

Na Tabela 1 são apresentados para cada amostra os índices físicos da mistura solo-cimento após a cura, a velocidade de deformação do ensaio e a resistência obtida. Os ensaios apresentados na Tabela 1 foram realizados em amostras moldadas com teor de cimento de $5 \%$. Os resultados dos ensaios são mostrados nas Figura 8 e 9 em termos de peso específico seco e índice de vazios. 
Tabela 1 - Variação da velocidade de deformação. *

\begin{tabular}{|c|c|c|c|c|c|}
\hline Ensaio & $\begin{array}{c}\text { Velocidade } \\
\mathbf{m m} / \mathbf{m i n}\end{array}$ & $* * \mathbf{w}_{\mathbf{p c}} \mathbf{( \% )}$ & $\begin{array}{c}* * * \gamma_{\mathbf{d}} \\
\mathrm{kN} / \mathrm{m}^{3}\end{array}$ & $\begin{array}{c}* * * * \\
\mathbf{S}(\mathbf{\%})\end{array}$ & $\begin{array}{c}\text { Resistênica } \\
\mathbf{( M p a )}\end{array}$ \\
\hline CP 01 & 0,01 & 0,67 & 16,94 & 3,06 & 0,75 \\
\hline CP 02 & 0,01 & 0,49 & 17,20 & 2,31 & 1,00 \\
\hline CP 03 & 0,01 & 0,68 & 17,95 & 3,65 & 1,50 \\
\hline CP 04 & 0,01 & 0,47 & 17,53 & 2,35 & 0,60 \\
\hline CP 05 & 0,01 & 0,38 & 17,37 & 1,85 & 0,50 \\
\hline CP 06 & 0,01 & 0,59 & 18,13 & 3,24 & 2,90 \\
\hline CP 07 & 0,01 & 0,70 & 18,08 & 3,84 & 2,65 \\
\hline CP 08 & 0,01 & 0,97 & 18,74 & 5,95 & 5,45 \\
\hline CP 09 & 1,00 & 0,29 & 17,19 & 1,36 & 0,75 \\
\hline CP 10 & 1,00 & 0,29 & 17,80 & 1,52 & 1,00 \\
\hline CP 11 & 1,00 & 1,03 & 17,12 & 4,80 & 0,85 \\
\hline CP 12 & 1,00 & 0,72 & 17,12 & 3,37 & 0,55 \\
\hline CP 13 & 1,00 & 0,24 & 17,67 & 1,21 & 0,80 \\
\hline CP 14 & 1,00 & 0,96 & 18,20 & 5,36 & 4,20 \\
\hline CP 15 & 1,00 & 0,86 & 18,26 & 4,87 & 4,20 \\
\hline CP 16 & 1,00 & 0,64 & 18,45 & 3,75 & 3,50 \\
\hline
\end{tabular}

*(teor de cimento das amostras - 5\%)

${ }^{* *} \mathrm{w}_{\mathrm{pc}}(\%)$ - umidade pós-cura***$\gamma_{\mathbf{d}}$ - peso específico aparente seco

****s \% - grau de saturação das amostras

\section{Citar $\gamma_{\mathrm{d} \text { e }} \%$}

Nos gráficos das Figuras 8 e 9 verifica-se a mesma tendência observada nos resultados anteriores em relação ao aumento da resistência com o aumento da intensidade de compactação.

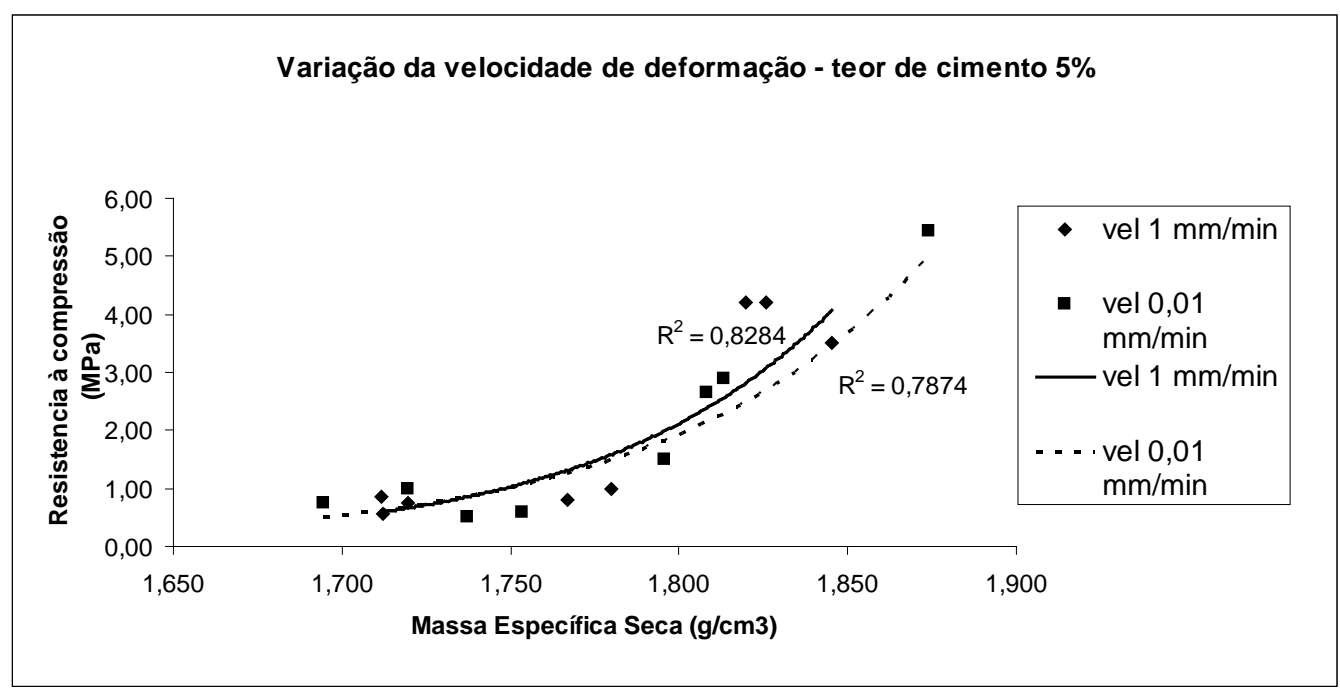

Figura 8 - Efeito da variação da velocidade de deformação sobre a relação resistência à compressão x massa específica seca. 


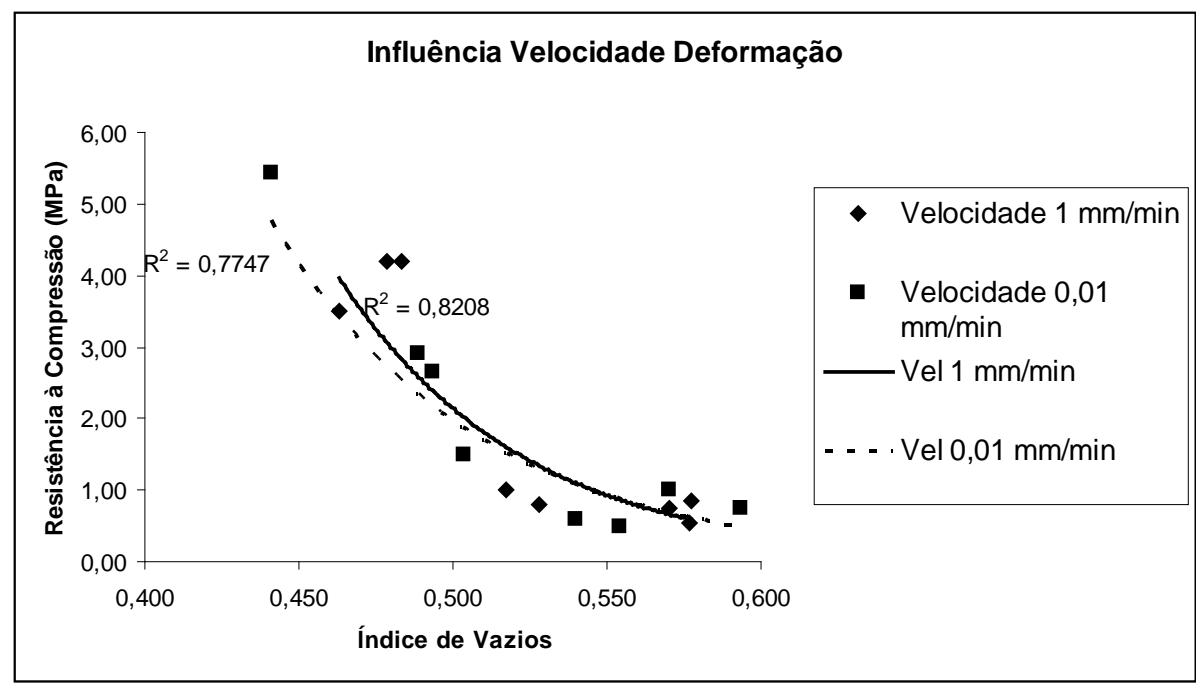

Figura 9 - Efeito da velocidade de deformação sobre a relação resistência à compressão $x$ índice de vazios.

No que diz respeito ao efeito da velocidade de deformação, observa-se que para o teor de cimento (5\%) ocorre uma tendência de aumento da resistência mecânica em função do aumento da velocidade de deformação sendo mais evidenciada essa tendência para menores valores dos índices de vazios (abaixo de 0,5).

\section{Criar parágrafo que faça uma ligação para as conclusões ou considerações finais.} No presente trabalho foram analisados os efeitos do teor de cimento, da densidade, do umedecimento e da velocidade de deformação na resistência à compressão simples em amostras cimentadas de solo proveniente da Formação Barreiras. As conclusões serão elencadas a seguir.

\section{CONCLUSÕES}

O Estudo mostrou que o solo analisado apresenta aumento da resistência a compressão com o aumento do teor de cimento. Esse aumento é significativo para solos muito compactos (baixos índices de vazios) e tem menor importância para valores mais elevados de índice de vazios. Verifica-se que, para um dado teor de cimento, a resistência diminui exponencialmente com o aumento do índice de vazios do solo (ou aumenta com a energia de compactação) tanto para as amostras inundadas como para as amostras secas, confirmando os estudos de Consoli et al. (2007), no qual demonstraram que para os teores de cimento, por eles estudados, em amostras na umidade natural, a resistência à compressão simples cresce linearmente com o aumento do teor de cimento e exponencialmente com a redução da porosidade da mistura compactada.

Para baixos teores de cimento (2\%) as amostras inundadas apresentam resistência inferior àquelas obtidas nos ensaios secos, ou seja, a diminuição da sucção provocada pela inundação das amostras provoca redução da resistência.

Os resultados para 5\% de cimento mostraram uma tendência mais efetiva do crescimento da resistência com o aumento da energia de compactação comparativamente com os mostrados nas amostras com $2 \%$ de cimento. Por outro lado, a sucção parece afetar menos a resistência dos solos com 5\% de cimento, principalmente para maiores índices de vazios e o 
umedecimento não afetou a resistência das amostras com $10 \%$ de cimento. Nesse caso o efeito da cimentação é mais importante que o da sucção no comportamento do solo.

Para amostras estudadas com teor de cimento de $5 \%$ e velocidades de deformação de 1,0 $\mathrm{mm} / \mathrm{minuto}$ e $0,01 \mathrm{~mm} / \mathrm{minuto}$ observou-se que ocorre uma tendência de aumento da resistência mecânica em função do aumento da velocidade de deformação para maiores valores de massa específica seca, acima de $1,80 \mathrm{~g} / \mathrm{cm}^{3}$.

A utilização do cimento Portland no melhoramento das propriedades do solo é uma alternativa bastante utilizada em Geotecnia. A aplicação desta técnica de tratamento de solos com cimento vem sendo empregada com sucesso para proteção de taludes em barragens de terra e canais, na contenção de plumas de contaminação, na construção de bases para pavimentos, melhoria de camada suporte para fundações rasas ou ainda no encapsulamento de solos contaminados. Entretanto, ainda não existem metodologias de dosagem racionais para utilização das misturas solo-cimento e o uso de solos lateríticos melhorados com cimento se mostra uma alternativa bastante eficaz para as diversas aplicações apresentadas.

\section{AGRADECIMENTOS}

Os autores gostariam de expressar seu agradecimento ao $\mathrm{CNPq}$ pela concessão da bolsa PIBUTI a um dos seus autores, bem como ao IFRN pelo apoio para a realização deste trabalho.

Este trabalho tem o suporte do Programa PIBITI do CNPq Brasil, pelo apoio financeiro.

\section{REFERÊNCIAS}

1- ABNT. (1991). NBR 11.578 - Cimento Portland Composto. Rio de Janeiro, Brasil.

2- ABNT. (1990). NBR 12.025 - Solo-Cimento: Ensaios de compressão simples de corposde-prova cilíndricos. Rio de Janeiro, Brasil.

3- Clough, G. W.; Sitar, N.; Bachus, R. C.; Rad, N. S. (1981): Cemented Sands under Static Loading. Journal of the Geotechnical Engineering Division, ASCE, Vol 107, N. 6, pp 799817.

4- Consoli, N. C.; Foppa, D.; Festugato L.; Heineck, K. S. Key. (2007). Key Parameters for Strenght Control of Articialy Cemented Soils. Journal of Geotechnical and Geoenvinmental Engineering. ASCE, Vol. 133, N.2, pp. 197 - 205.

5- Lo, S. R.; Wardani, S. P. R. (2002): Strength and Dilatancy of a Silt Stabilized by a Cement and Fly Ash Mixture. Canadian Geotechnical Journal, Vol. 39, N. 1, pp. 77-89.

6- Pinto, C. de S. (2000). Curso Básico de Mecânica dos solos. Oficina de Textos, São Paulo. $226 \mathrm{p}$.

7- Santos Júnior, O. F.; Scudelari, A. C.; Amaral, R. F. (2004). Geotechnical Properties of Tertiary Sediments and Failure Mechanisms of a Sea Cliff in the State of Rio Grande do Norte, North East Brazil. IV International Symposium on Landslides, Rio de Janeiro, Brasil, v. 1, 729-733 p.

8- Schnaid, F.; Prietto, P. D. M.; Consoli, N. C. (2001): Characterization of Cemented Sand in Triaxial Compression. Journal of Geotechnical and Geoenvironmental Engineering, ASCE, Vol 127, N. 10, pp. 857-868. 
9- Santos Júnior, O. F.; Severo, R. N. F.; Freitas Neto, O.; França, F. A. N. (2005). Análise da Estabilidade nas Falésias entre Tibau do Sul e Pipa - RN. IV Conferência Brasileira Sobre Estabilidade de Encostas, Salvador, Brasil, v.I , 487-496 p.

10- Severo, R. F. (2005). Análise da Estabilidade das Falésias entre Tibau do Sul e Pipa $R N$. Dissertação de Mestrado, Universidade Federal do Rio Grande do Norte, Natal, Brasil. 11- Silva, W. S. (2003) Estudo da dinâmica superficial e geotécnica das falésias do município de Tibau do Sul - Litoral Oriental do RN. Dissertação de Mestrado, Universidade Federal do Rio Grande do Norte, Natal, Brasil. 